\title{
GENERALISED MARKOVIAN CONTROL SYSTEMS
}

\author{
P. E. KLOEDEN
}

(Received 30 July 1973)

Communicated by B. Mond

\section{Introduction}

The qualitative behaviour of control systems based on ordinary differential equations has been investigated with clarity and elegance using axiomatically defined General Control Systems. Here an attainablity set function, evolving in semigroup fashion, is the main entity of interest [1], [2], [3], [4].

In stochastic control theory quite complicated and messy mathematical machinery is required, usually obscuring the properties being investigated. It is felt that an analogous axiomatic approach could help circumvent this difficulty, at least for stochastic systems with perfect state observations and satisfying the Markov Principle, i.e. the future evolution is completely determined by the dynamics of the system and the current state, independently of how the current state was attained.

In [5] the author showed that the attainability set function of stochastic control systems, governed by Itô stochastic equations and with perfect state observations, had similar properties to the attainability sets of deterministic systems. There the state space was a space of random variables with the topology of mean square convergence. However, any axiomatic attempt in this space would require additional measurability axioms. To avoid this it is necessary to consider probability measures as the basic state points. Then the attainability set function satisfies all but one (a minor one) of the axioms of a deterministic General Control System. The price paid for this simplification is the use of a considerably weaker topology, that of weak convergence of probability measures. The advantages though are quite tempting: the underlying space on which the probability measures are defined can be either finite or a continuum; there is need to specify the source or sources of randomisation, thus avoiding many of the modeling difficulties associated with stochastic control systems; the extensive qualitative theory developed for deterministic General Control Systems carries over with little modification. 
In this note we will axiomatically define a Generalised Markovian Control System on a space of probability measures. A simple example involving finite dimensional transition matrices will be given. The note will conclude with some comments on the stability results applicable to the system.

Unfortunately this approach does not seem to be extendable to include random times, imperfect observations or non-Markovian processes (that is, those not satisfying the Markov principle.)

\section{Notation and preliminaries}

Let $(X, d)$ be a metric space and $\mathscr{B}$ the collection of all Borel subsets on $X$. Denote by $B(X)$ the set of all realvalued bounded $\mathscr{B}$-measurable functions on $X, C(X)$ the subset of $B(X)$ consisting of all functions on $X$, and $V(\mathscr{B})$ the set of all finite realvalued countably additive set functions on $\mathscr{B}$. Then

$$
\mathscr{X}=\{\mu \in V(\mathscr{B}) ; \mu(A) \geqq 0 \quad \forall A \in \mathscr{B}, \mu(X)=1\}
$$

is the set of probability measures defined on $X$.

We will put on $\mathscr{X}$ the topology of weak convergence, as it is called in standard probabilistic terminology.

Let $C^{*}(X)$ denote the dual of $C(X)$. Then $V(\mathscr{B}) \cong C^{*}(X)$ by a Riesz Representation Theorem. Let $V(\mathscr{B})$ have the weak ${ }^{*}$ topology. Then the induced relative topology on $\mathscr{X}$ is the topology of weak convergence.

Since $\mu \in V(\mathscr{B})$ can be represented uniquely as a linear functional $L_{\mu}$ on $C(X)$, namely $L_{\mu}(f)=\int_{X} f d \mu$, then a sequence $\mu_{n} \in \mathscr{X}$ converges weakly to $\mu \in \mathscr{X}$ if and only if for each $f \in C(X)$

$$
\lim _{n \rightarrow \infty}\left|\int_{X} f d \mu_{n}-\int_{X} f d \mu\right|=0
$$

The following well known theorem will be needed.

THeOREM ([7], [8]. [9]). $\mathscr{X}$ is a compact metric space if and only if $X$ is compact metric.

In this note we will assume that the space $X$, in which the control system is acting, is compact and metric. This is true for finite spaces $X=(1,2, \cdots, N)$ and holds for the systems considered in [5], provided they are restricted with probability 1 (w.p. 1) to a compact subset of $\mathbb{R}^{\prime \prime}$. Consequently $\mathscr{X}$ will be compact and metric. Compatible metrics are given by Kallianpur [8] and Prokhorov [9]. Such a metric will be denoted $\rho$.

When $X=(1,2, \cdots, N)$

$$
\mathscr{X}=\left\{p \in \mathbb{R}^{N} ; p_{i} \geqq 0, i=1,2, \cdots, N \text { and } \sum_{i=1}^{N} p_{i}=1\right\} .
$$


Here the topology of weak convergence is the subspace topology induced by the usual topology on $\mathbb{R}^{N}$ and $\mathscr{X}$ is obviously a compact subspace of $\mathbb{R}^{N}$.

For nonempty subsets $A, B$ of $\mathscr{X}$ define

$$
\begin{aligned}
& \rho(\mu, B)=\inf _{\phi \in B} \rho(\mu, \phi) \quad \text { where } \mu \in \mathscr{X} \\
& \rho^{*}(A, B)=\sup _{\mu \in A} \rho(\mu, B)
\end{aligned}
$$

and

$$
\rho(A, B)=\max \left\{\rho^{*}(A, B), \rho^{*}(B, A)\right\} \text { the Hausdorff metric on }
$$

nonempty closed (hence compact) subsets of $\mathscr{X}$.

\section{Definition of a Generalised Markovian Control System}

A Generalised Markovian Control System (g.m.c.s.) on $(\mathscr{X}, \rho)$ will be given in terms of an attainability set function $F\left(\mu_{0}, t_{0}, t\right)$, the set of all $\mu \in \mathscr{X}$ that can be reached from the initial position $\left(\mu_{0}, t_{0}\right) \in \mathscr{X} \times \mathbb{R}^{+}$at time $t \geqq t_{0}$ under all possible choices of the control action. The attainability set function is assumed to satisfy the following axioms:

(I) $F\left(\mu_{0}, t_{0}, t\right)$ is a compact nonempty subset of $\mathscr{X}$, defined for every $\mu_{0} \in \mathscr{X}$ and $0 \leqq t_{0} \leqq t<\infty$,

(II) Initial condition: $F\left(\mu_{0}, t_{0}, t_{0}\right)=\left\{\mu_{0}\right\}$ for all $\mu_{0}, t_{0}$,

(III) Semigroup evolution: for all $\mu_{0}$ and $t_{0} \leqq t_{1} \leqq t_{2}$

$$
F\left(\mu_{0}, t_{0}, t_{2}\right)=\bigcup\left\{F\left(\phi_{1}, t_{1}, t_{2}\right) ; \phi_{1} \in F\left(\mu_{0}, t_{0}, t_{1}\right)\right\}
$$

(IV) $F\left(\mu_{0}, t_{0}, t\right)$ is continuous in $t$ : given $\mu_{0}, t_{0} \leqq t_{1}, \varepsilon>0$ there exists a $\delta>0$ such that

$$
\rho\left(F\left(\mu_{0}, t_{0}, t\right), F\left(\mu_{0}, t_{0}, t_{1}\right)\right)<\varepsilon \quad \text { for }\left|t-t_{1}\right|<\delta
$$

(V) $F\left(\mu_{0}, t_{0}, t\right)$ is upper semicontinuous in $\left(\mu_{0}, t_{0}\right)$ uniformly in any finite interval $t_{1} \leqq t \leqq t_{1}$ : given $\mu_{0}, 0 \leqq t_{0} \leqq t_{1} \leqq t_{2}, \varepsilon>0$ there exists a $\delta>0$ such that

$$
\begin{aligned}
& \rho^{*}\left(F\left(\mu_{0}^{\prime}, t_{0}^{\prime}, t\right), F\left(\mu_{0}, t_{0}, t\right)\right)<\varepsilon \quad \text { for all } \mu_{0}, t_{0}, t \text { satisfying } \\
& \rho\left(\mu_{0}^{\prime}, \mu_{0}\right)<\delta,\left|t_{0}^{\prime}-t_{0}\right|<\delta, t_{1} \leqq t \leqq t_{2} \text { and } 0 \leqq t_{0}^{\prime}, t_{0} \leqq t
\end{aligned}
$$

Axioms I and III imply

(a) the system satisfies the Markov Principle

(b) the class of admissible control functions is sufficiently large to include piecewise combinations of admissible controls, and is compact in some sense (see [5]). 
Deterministic General Control Systems usually also satisfy a backwards extendability axiom: given $\mu_{1}, t_{0} \leqq t_{1}$ there is a $\mu_{0}$ such that $\mu_{1} \in F\left(\mu_{0}, t_{0}, t_{1}\right)$. This is not so for stochastic systems. Its omission introduces start events into the system, the consequences of which are discussed in [6].

Definition. A trajectory of a g.m.c.s. is a mapping $\phi:\left[t_{0}, t_{1}\right] \rightarrow \mathscr{X}$ such that $\phi\left(t_{b}\right) \in F\left(\phi\left(t_{a}\right), t_{a}, t_{b}\right)$ for all $t_{0} \leqq t_{a} \leqq t_{b} \leqq t_{1}$.

The following results are proved in [6] for General Control Systems defined on a locally compact metric space and satisfying axioms I to $\mathrm{V}$.

THEOREM. Each trajectory of a g.m.c.s. is continuous in $t$.

THEOREM. If for a certain g.m.c.s. $\mu_{1} \in F\left(\mu_{0}, t_{0}, t_{1}\right)$, then there exists a trajectory $\phi(t)$ defined on $\left[t_{0}, t_{1}\right]$ such that $\phi\left(t_{0}\right)=\mu_{0}$ and $\phi\left(t_{1}\right)=\mu_{1}$.

We also require these trajectories to be meaningful in some probabilistic sense as stochastic processes. To do this we introduce the following definition due to Neveu ([10], page 73.$)$

Definition. Given two measurable spaces $\left(X, \mathscr{A}_{1}\right)$ and $\left(X_{2}, \mathscr{A}_{2}\right)$, a transition probability $P_{2}^{1}$ is a mapping of $X_{1} \times \mathscr{A}_{2}$ into $[0,1]$ such that:

(a) for every $x_{1} \in X_{1}, P_{2}^{1}\left(x_{1}, \cdot\right)$ is a probability measure on $\left(X_{2}, \mathscr{A}_{2}\right)$.

(b) for every $A \in \mathscr{A}_{2}, P_{2}^{1}(\cdot, A)$ is measurable on $\left(X, \mathscr{A}_{1}\right)$.

Then the following result holds:

THEOREM. Each trajectory $\phi(t)$ of a g.m.c.s. on $\left[t_{0}, t_{1}\right]$ is a transition probability in the sense of Neveu.

Proof. Let

$$
\begin{aligned}
& X_{1}=\left[t_{0}, t_{1}\right] \text { the interval of definition of } \phi(t) \\
& \mathscr{A}_{1}=\text { the Borel field on }\left[t_{0}, t_{1}\right] \\
& X_{2}=X \\
& \mathscr{A}_{2}=\mathscr{B} \text { the Borel field on } X .
\end{aligned}
$$

Then (a) follows since $\phi(t)$ is a probability measure on $X$ for each $t \in\left[t_{0}, t_{1}\right]$ and (b) follows from the continuity of $\phi(t)$ in $t$.

Although the g.m.c.s. satisfies the Markov Principle, its trajectories need not be Markov processes in the strict probabilistic sense. In the example to be given the trajectories are actually piecewise Markov processes, but not Markov processes. Piecewise Markov processes, just recently introduced by Kuczura [11], are actually more realistic in control theory and result from the switching of controls. 


\section{An example}

Here we give a simple example of a g.m.c.s. based on an even simpler stochastic control system defined on a finite state space $X=\{1,2, \cdots, N\}$. It is autonomous, that is, stationary in probabilistic terminology.

Let $P(t)$ be a standard $N \times N$ Markov transition matrix i.e. satisfying

(i) $P(0)=I$ identity matrix

(ii) $P(s+t)=P(s) P(t)$ for any $s, t \geqq 0$

(iii) $P_{i j} \geqq 0$ for $i, j=1,2, \cdots, N$

(iv) $\sum_{j=1}^{N} P_{i j}=1$ for $i=1,2, \cdots, N$

(v) $P(t)$ is continuous in $t$, for all $t \geqq 0$.

We will define an attainability set function

by

$$
F: \mathscr{X} \times \mathbb{R}^{+} \rightarrow 2^{\mathscr{X}}
$$

$$
F\left(p_{0}, t\right)=\bigcup_{0 \leqq s \leqq t}\left\{P(s) p_{0}\right\} \text { for all } t \geqq 0, p_{0} \in \mathscr{X}
$$

This corresponds to a control system with only two control actions: apply $P(t)$ or leave it switched off (i.e. apply the identity.)

The following five propositions show that the attainability set function satisfies the axioms of a g.m.c.s.

Proposition. $F\left(p_{0}, t\right)$ is defined for all $p_{0} \in \mathscr{X}$ and $t \geqq 0$, for which it is a nonempty compact subset of $\mathscr{X}$.

Proof. This follows from the definition, the continuity of $P(t)$ and the compactness of $[0, t]$.

Proposition. $F\left(p_{0}, 0\right)=\left\{p_{0}\right\}$ for all $p_{0} \in \mathscr{X}$.

PROPOSITION. $F\left(p_{0}, t+s\right)=F\left(F\left(p_{0}, t\right), s\right)$ for all $s, t \geqq 0, p_{0} \in \mathscr{X}$.

Proof.

$$
\begin{gathered}
F\left(p_{0}, t+s\right)=\bigcup\left\{P(\tau) p_{0} ; 0 \leqq \tau \leqq t+s\right\}=\bigcup\left\{P\left(\tau_{1}+\tau_{2}\right) p_{0} ; 0 \leqq \tau_{1} \leqq t,\right. \\
\left.0 \leqq \tau_{2} \leqq s\right\} \\
=\bigcup\left\{P\left(\tau_{2}\right) P\left(\tau_{1}\right) p_{0} ; 0 \leqq \tau_{1} \leqq t, 0 \leqq \tau_{2} \leqq s\right\}=\bigcup\left\{P\left(\tau_{2}\right) q ; 0 \leqq \tau_{2} \leqq s,\right. \\
q \in F\left(p_{0}, t\right)=F\left(F\left(p_{0}, t\right), s\right) .
\end{gathered}
$$

Proposition. $F\left(p_{0}, t\right)$ is uppersemicontinuous in $\left(p_{0}, t\right)$.

Proof. Suppose this is not so. Then there exist $p_{n} \rightarrow p_{0}, t_{n} \rightarrow t$ and $q_{n} \in F\left(p_{n}, t_{n}\right)$ such that $\left\{q_{n}\right\}$ has no limit point in $F\left(p_{0}, t\right)$.

Now $\mathscr{X}$ is compact, so $\left\{q_{n}\right\}$ has limit points. Also there are $\left\{\tau_{n}\right\}$ with $0 \leqq \tau_{n} \leqq t_{n}$ such that $q_{n}=P\left(\tau_{n}\right) p_{n}$. Since $t_{n} \rightarrow t$ we have $t_{n}, t \in[0, T]$ for some $T<\infty$. Then $\tau_{n} \in[0, T]$ for each $n$, and consequently has a convergent subsequence $\tau_{m}^{\prime} \rightarrow \tau_{0}$, where $\tau_{0} \in[0, T]$. 
Let $q_{m}^{\prime}=P\left(\tau_{m}^{\prime}\right) p_{m}^{\prime}$. Then by the continuity of the function

$$
g(s, p)=P(s) p, q_{m}^{i} \rightarrow P\left(\tau_{0}\right) p_{0}=q_{0},
$$

and thus the hypothesis is contradicted.

Proposition. $F\left(p_{0}, t\right)$ is continuous in $t$.

Proof. In view of the preceding proposition it remains only to prove that

$$
\lim _{t \rightarrow t_{0}} \rho^{*}\left(F\left(p_{0}, t_{0}\right), F\left(p_{0}, t\right)\right)=0
$$

Since $F\left(p_{0}, t_{0}\right)$ is compact this is equivalent to showing that for any $q_{0} \in F\left(p_{0}, t\right)$ and $t_{n} \rightarrow t_{0}$ there is a sequence $q_{n} \in F\left(p_{0}, t_{n}\right)$ such that $q_{n} \rightarrow q_{0}$ as $n \rightarrow \infty$.

We will construct such a sequence. Since $q_{0} \in F\left(p_{0}, t_{0}\right)$ there is a $\tau_{0} \in\left[0, t_{0}\right]$ such that $q_{0}=P\left(\tau_{0}\right) p_{0}$. Then define $\left\{q_{n}\right\}$ as follows:

(i) $0 \leqq \tau_{0} \leqq t_{0} \leqq t_{n}$, $q_{n}=P\left(t_{n}-t_{0}+\tau_{0}\right) p_{0}$

(ii) $0 \leqq \tau_{0} \leqq t_{n} \leqq t_{0}$, $q_{n}=q_{0}=P\left(\tau_{0}\right) p_{0}$,

(iii) $0 \leqq t_{n} \leqq \tau_{0} \leqq t_{0}$, $q_{n}=P\left(t_{n}\right) p_{0}$.

In each case $q_{n} \in F\left(p_{0}, t_{n}\right)$ and clearly $q_{n} \rightarrow q_{0}$ as $t_{n} \rightarrow t_{0}$.

We now give an example of a trajectory of this g.m.c.s. which is not a Markov process.

Define

$$
S(t)=\left\{\begin{array}{l}
l \text { for } 0 \leqq t \leqq 1 \\
P(t-1) \text { for } t \geqq 1
\end{array}\right.
$$

Then for any $p_{0} \in \mathscr{X}, \phi(t)=S(t) p_{0}$ is a trajectory of the g.m.c.s, but it is not a Markov process as $S(t)$ does satisfy the semigroup relation required of Markov transition matrices. It is however a piecewise Markov process, according to the definition of Kuczura.

\section{Stability in a G.M.C.S.}

The stability theory of Generalised Markovian Control Systems is a special case of that for General Control Systems without the backwards extendability axiom. This is discussed in [6].

There are two basic concepts: strong properties if every trajectory satisfies the desired property, and weak if at least one trajectory satisfies the property. From the control theoretic viewpoint the weak properties are of greater interest.

Many types of stability can be considered e.g. stability, asymptotic stability, finite time stability, etc., in both strong and weak cases. For example

DEFINITION. $A$ set $A \subset \mathscr{X}$ is said to be strongly stable (with respect to a certain g.m.c.s.) if for every $t_{0} \geqq 0$ and $\varepsilon>0$ there is a $\delta=\delta\left(\varepsilon, t_{0}\right)>0$ such that 


$$
F\left(S_{\rho}(A), t_{0}, t\right) \subset S_{e}(A) \text { for all } t \geqq t_{0}
$$

(Here $\left.S_{\rho}(A)=\{\mu \in \mathscr{X} ; \rho(\mu, A)<\delta\}\right)$.

Definition. $A$ set $A \subset \mathscr{X}$ is said to be weakly stable if for every $t_{0} \geqq 0$ and $\varepsilon>0$ there is a $\delta=\delta\left(t_{0}, \varepsilon\right)>0$ such that for each $p_{0}$ with $\rho\left(p_{0}, A\right)<\delta$ there exists a trajectory $\phi(t)$ with $\phi\left(t_{0}\right)=p_{0}$ and satisfying

$$
\rho(\phi(t), A)<\varepsilon \text { for all } t \geqq t_{0} .
$$

Necessary and sufficient conditions in terms of Liapunov functions have been given by Roxin and Zubov ([1], [2], [3], [4]) for many kinds of stabilities of a set. These will carry over to the g.m.c.s., with the modifications mentioned in [6].

Szegö and Trecanni [12] have developed a theory on limit sets for General Control Systems, and should be of interest from a probabilistic point of view.

\section{References}

[1] E. O. Roxin, 'Stability in General Control Systems', J. Diff. Equat. 1 (1965), 115-150.

[2] E. O. Roxin, 'On Stability in Control Systems', J. SIAM Control 3 (1966), 357-372.

[3] E. O. Roxin, 'On Asymptotic Stability in Control Systems', Rend. Circ. Matem. Palermo Serie II, Tomo XV (1966).

[4] E. O. Roxin, 'On Finite Stability in Control Systems', Rend. Circ. Matum. Palermo Serie II, Tomo XV (1966).

[5] P. E. Kloeden, Attainability Sets of Stochastic Control Systems, (University of Queensland Pure Maths. Preprint 1973.)

[6] P. E. Kloeden, General Control Systems Without Backwards Extension, in proceedings of Conf. on Differential Games and Control Theory, (Univ. of Rhode Island), June 4-8 1973; P. T. Liu and E. O. Roxin (ed.); Marcel-Dekker (1973).

[7] K. R. Parthasarthy, Probability Measures on Metric Spaces, (Academic Press, New York and London 1967).

[8] Yu. V. Prokhorov, 'Convergence of Stochastic Processes and Limit Theorems', Theory of Probability and Applications 1 (1956), 157-214.

[9] G. Kallianpur, 'The Topology of Weak Convergence of Probability Measures', J. Math. and Mech. Vol. 10 (1961), 947-969.

[10] J. Neveu, Mathematical Foundations of the Calculus of Probability, Holden-Day Inc. San Francisco (1965).

[11] A. Kuczura, 'Piecewise Markov Processes', SIAM. J. Appl. Math. 24, (1973), 169-184.

[12] G. Szegö and G. Trecanni, Semigruppi di Transformatzioni Multivoche, Lecture Notes in Mathematics No. 101, Springer-Verlag (1969).

Department of Mathematics

University of Queensland

Australia 